\title{
3.5 Prevalence of Hypertension and Urinary Abnormalities in an Indian
}

\section{Paediatric Population}

A. Aiello (1), M. Gallieni (2), M. Giussani (3), S.K. Brahmochary Mandal (4),

F. Pieruzzi (1), A. Stella (1), S. Genovesi (1)

(1)Università Milano-Bicocca, Milano, Italy; (2)Ospedale San Paolo, Milano, Italy;

(3)FIMP, Milano, Italy; (4)Institute For Indian Mother And Child, Kolcata, India

Introduction. Hypertension and nephropathies are becoming a major problem even in low-income countries, but data relating to child population are limited. In high-income countries paediatric hypertension is associated with overweight $(\mathrm{OW})$ and obesity $(\mathrm{OB})$, conditions rarely found in poor countries.

Aim. To determine the prevalence of hypertension and/or urinary abnormalities in children living in a rural area south of Kolcata.

Methods. We measured arterial blood pressure and tested the presence of haematuria and/or proteinuria (Comburtest, Roche) in 1200 children between 5-12 years (average age $8.35 \pm 1.4,592$ males). Children were defined hypertensive when values of systolic (SBP) and/or diastolic blood pressure (DBP) were $>95$ th percentile and pre-hypertensive those with values of SBP and/or DBP $>$ 90th and $<95$ th percentile according to age, gender and height. OW and OB children were identified by the International Obesity Task Force standards.

Results. Prevalence of hypertension was $3.0 \%$, without differences in the two genders, $0.8 \%$ were pre-hypertensive. Prevalence of excess weight was low: $\mathrm{OW}=1.4 \%$ and $\mathrm{OB}=0.4 \%$. Prevalence of hypertension in normal weight (NW) subjects was significantly lower than that of OW or OB children $(\mathrm{NW}=2.7 \%, \mathrm{OW}=23 \%, \mathrm{OB}=25 \%, \mathrm{p}<0.001)$. The percentage of subjects with hypertension was similar in the two genders in the NW, but between excess weight children the hypertension prevalence was slightly higher in females $(4 / 13 \mathrm{~F}$ vs $0 / 9 \mathrm{M}, \mathrm{p}=0.06)$. Microhaematuria was present in 124 subjects $(10.3 \%)$, only one of which was also hypertensive. Proteinuria was found in 25 children $(2.1 \%)$, none of whom had hypertension. In 6 subjects there were both microhaematuria and proteinuria $(0.5 \%)$.

Conclusions. (i) even if the excess weight of the sample studied is low, the prevalence of hypertension is similar to that of high income countries; (ii) when present, excess weight is significantly associated with hypertension; (iii) the prevalence of urinary abnormalities is high, although not associated with hypertension. Our data suggest a high prevalence of primary hypertension in Indian children. Alternatively, unidentified factors conditioning hypertension might be present in this population. 